\title{
Structure of a human pulmonary acinus
}

\author{
N Berend, A C Rynell, H E Ward
}

\begin{abstract}
The structure of the human pulmonary acinus has been described infrequently. The aim of the study was to determine the branching pattern of respiratory bronchioles and alveolar ducts in a human acinus from the peripheral part of the lung, where space constraints may have affected airway branching patterns. The lungs were obtained from an 18 year old victim of a motor vehicle accident and fixed in inflation under a pressure of $25 \mathrm{~cm} \mathrm{H}_{2} \mathrm{O}$. A block was cut from the lower edge of the right lower lobe and embedded in plastic. Serial sections were cut and the branching pattern of airways subtended by a terminal bronchiole were followed. The acinus was bounded on two sides by pleura and on the remaining sides by connective tissue septa. The terminal bronchiole divided into two respiratory bronchioles, each of which gave rise to four systems of alveolar ducts. Between successive systems of alveolar ducts the respiratory bronchioles continued as single airways, becoming progressively more alveolated towards the periphery but not subtending further branches of respiratory bronchioles. The duct systems became less complex towards the periphery, near to the edge of the lung. The total volume of the acinus was similar to that found in previous studies. This branching pattern has not been described previously in a human acinus.
\end{abstract}

The structure of the human pulmonary acinus, defined functionally as the largest lung unit in which all airways participate in gas exchange, ${ }^{1}$ has seldom been described. The results of such studies have yielded a picture of acinar structure of considerable variability, both in size and in the number of generations of branches. ${ }^{2-8}$ In part, this variability may be a reflection of methodological differences in the various studies, but true heterogeneity of acinar geometry is also possible. This was highlighted in a recent study by Haefeli-Bleuer and Weibel, ${ }^{9}$ in which a seven to eight fold difference in acinar volume was documented within one lobe of a lung. In general, however, the terminal bronchiole has been observed to subtend several generations of respiratory bronchioles, which in turn subtend several generations of alveolar ducts.

In this study we sectioned an acinus from a subpleural location serially, finding an acinar structure not previously described in a human lung.

\section{Methods}

The right lung of an 18 year old man who had died of head injuries was obtained at necropsy. A chest radiograph obtained before death was normal. The lung was weighed and inflated with air to a transpulmonary pressure of $25 \mathrm{~cm}$ $\mathrm{H}_{2} \mathrm{O}$ and the lung volume was obtained by water displacement. The lung was then fixed in inflation via the bronchus at a constant transpulmonary pressure of $25 \mathrm{~cm} \mathrm{H}_{2} \mathrm{O}$ with $10 \%$ buffered formalin for 72 hours. The lung volume was then remeasured.

Multiple blocks were cut from various parts of the lung. The acinus described in this paper was selected from the lower edge of the right lower lobe posterolaterally in the costophrenic angle and was bounded on two sides and the distal end by pleura. The block of tissue was dehydrated in graded ethanol at room temperature. Infiltration and embedding were achieved by means of an LKB 2218-500 Historesin embedding kit. The tissue was preinfiltrated in 1:1 99\% ethanol:infiltration solution for 12 hours to ensure complete penetration. The tissue was then transferred to pure infiltration solution and kept at $4{ }^{\circ} \mathrm{C}$ for one week, the solution being changed three times. During this period the tissue was also intermittently subjected to vacuum to remove air. The tissue was then embedded in $20 \times 20 \times 20 \mathrm{~mm}$ polyethylene mould for polymerisation and the block was kept on ice and under refrigeration. Holes were drilled through the block at each corner to enable accurate stacking of sections for reconstruction.

After completion of polymerisation serial 5 $\mu \mathrm{m}$ sections were cut with a D profile tungsten carbide steel knife. Sections were stained with $0.5 \%$ toluidine blue in $1 \%$ borax. Every fifth section was used for the reconstruction, additional sections being viewed where it was difficult to decide on branching patterns.

Sections were photographed and the terminal bronchioles, respiratory bronchioles, and alveolar ducts identified, labelled, and traced. The outlines were then retraced on a digitising tablet and the airways reconstructed by means of a three dimensional reconstruction program (PC-3D Multidimensional Computing, Durham, North Carolina, USA).

All dimensions were corrected by two shrinkage factors. Shrinkage during fixation was calculated from the known volumes before and after fixation of the whole lung. Shrinkage 


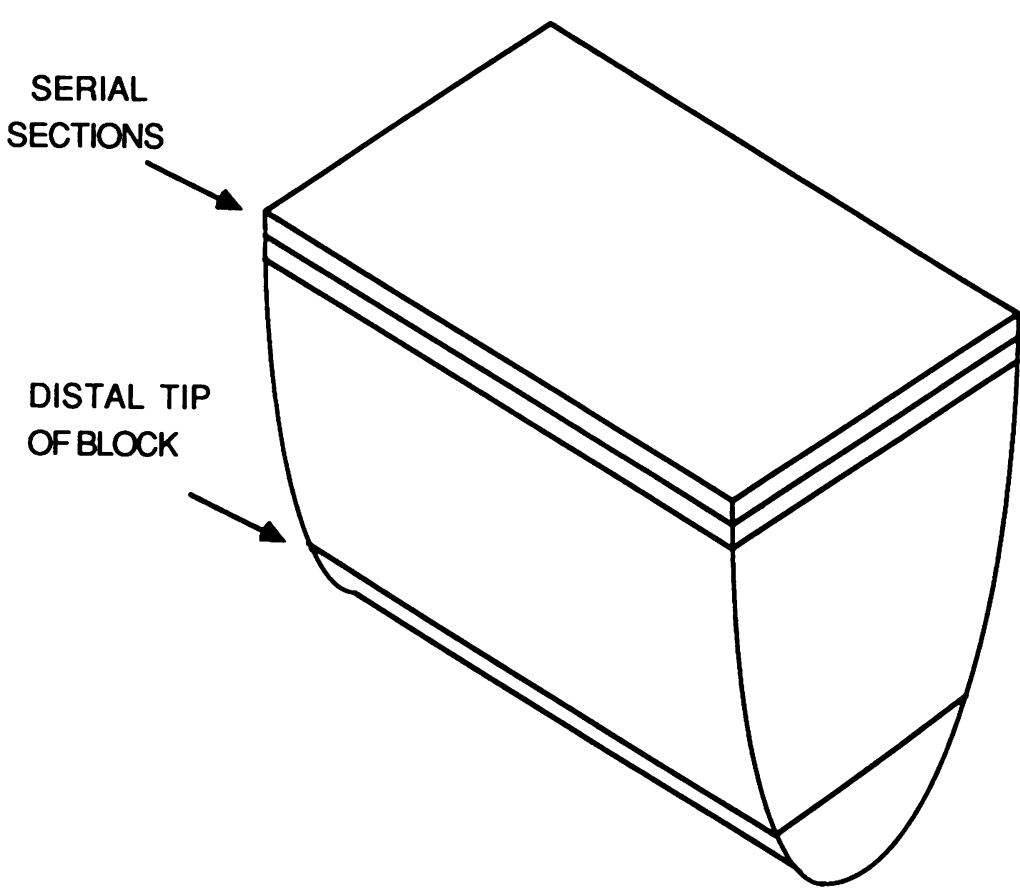

Figure 1 Configuration of the tissue block cut from the lower edge of the right lower lobe. Note that less than $1 \mathrm{~mm}$ could not be sectioned at the distal tip of the block.

Figure 2 Shape of the acinus in cross section, approximately that of a parallelogram bounded by two pleural surfaces and two connective tissue septa. The terminal bronchiole leading into the acinus is indicated. P-pleura: CTS - connective tissue septa; TB-terminal bronchiole.

\section{Results}

The weight of the right lung was $346 \mathrm{~g}$ and the air inflated volume $3701 \mathrm{ml}$. Volume shrinkage during fixation was $19 \%$ and area shrinkage during processing $4 \cdot 6 \%$. Serial sections were cut to within less than $1 \mathrm{~mm}$ of the pleural edge
Intervals along respiratory bronchioles between origins of alveolar duct systems

\begin{tabular}{lll}
\hline Interval $(\mu \mathrm{m})$ & $\begin{array}{l}\text { Left } \\
\text { hemiacinus }\end{array}$ & $\begin{array}{l}\text { Right } \\
\text { hemiacinus }\end{array}$ \\
\hline $\begin{array}{l}\text { Terminal bronchiole } \\
\text { bifurcation to 1st duct }\end{array}$ & 410 & 430 \\
system & & \\
1st to 2nd duct system & 870 & 630 \\
2nd to 3rd duct ysstem & 730 & $\begin{array}{l}\text { Overlap } \\
\text { 3rd to 4th duct system }\end{array}$ \\
\hline
\end{tabular}

at the distal extreme of the block of tissue (fig 1). Possibly therefore some distal branches were lost.

Reconstruction was facilitated by the enclosure of the acinus by two pleural surfaces and two connective tissue septa, producing a cross section the shape of a parallelogram (fig 2). The cross sectional area at this level was $45.6 \mathrm{~mm}^{2}$. The total length of the acinus was $3.82 \mathrm{~mm}$ without the missing tip. This is longer than the sum of the distances between the branches (table) because some of the first alveolar ducts recurved to be proximal to the bifurcation of the terminal bronchiole.

The terminal bronchiole before bifurcation showed some areas of flattened epithelium. The terminal bronchiole (fig 2) leading into the acinus divided into two respiratory bronchioles. The branch point is shown in figure 3 . All subsequent branches were alveolar ductsthat is, at each branch point there was one daughter respiratory bronchiole, other branches being alveolar ducts. The whole branching system of the acinus is shown schematically in figure 4. The first alveolar duct branches in the right hemiacinus (that is, the part of the acinus supplied by the right respiratory bronchiole) are shown in figure 5 and had their origin $430 \mu \mathrm{m}$ below the bifurcation of the terminal bronchiole. This was a combined

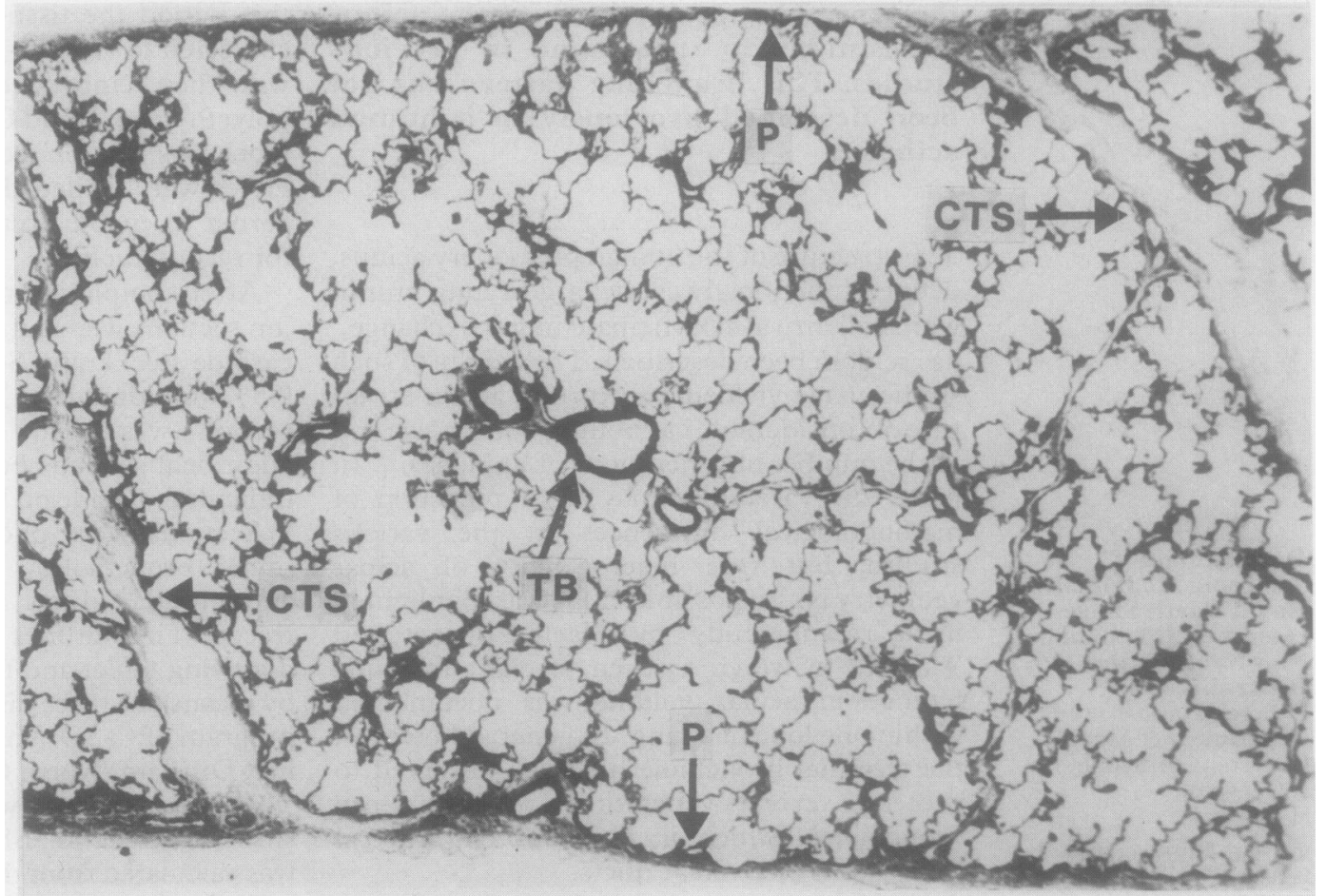




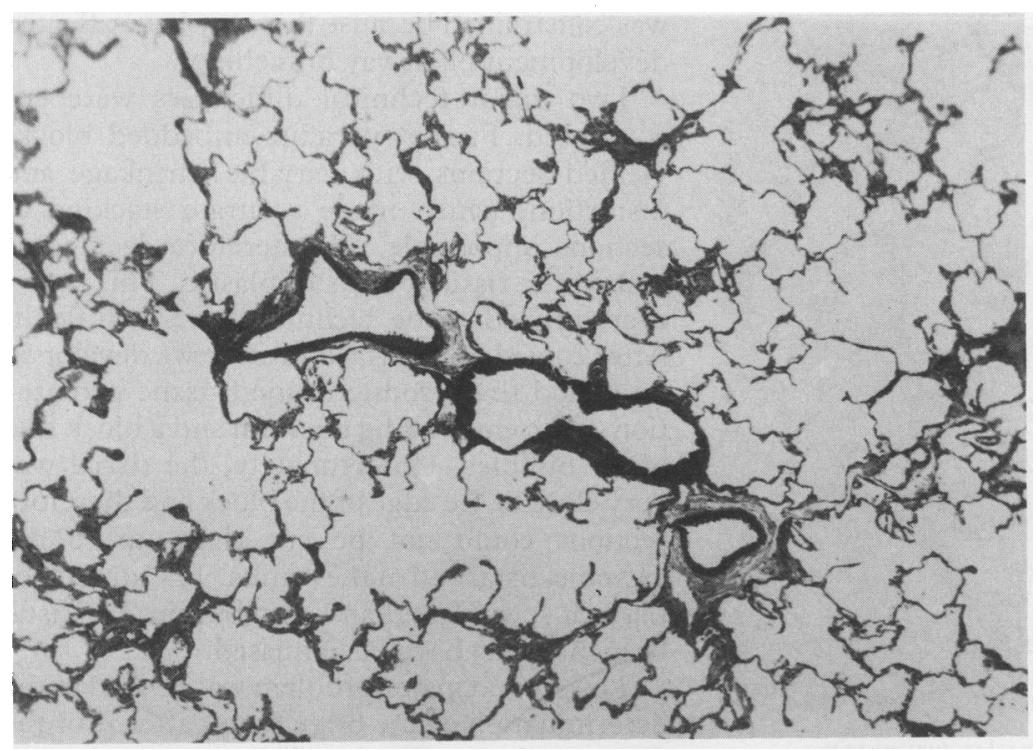

Figure 3 The terminal bronchiole about to divide into two respiratory bronchioles.

origin of three alveolar ducts, each of which subsequently gave rise to further ducts (fig 4). The respiratory bronchiole then continued as a single airway (fig 6) until, $630 \mu \mathrm{m}$ below the previous system of alveolar ducts, a second system arose. Over the same length of respiratory bronchiole giving rise to the second system a third system originated (fig 7), and this can be followed schematically in figure 4 . After a further interval of $650 \mu \mathrm{m}$ a final system originated, consisting of only two alveolar ducts with the respiratory bronchiole terminating in a fully alveolated state (fig 8).

A similar pattern was seen in the left hemiacinus (the portion of the acinus supplied by the left respiratory bronchiole). Again, the respiratory bronchiole continued as a single airway between duct systems with the distances between systems shown in the table. A final single duct arose $320 \mu \mathrm{m}$ below the third system and the respiratory bronchiole simply became fully alveolated and terminated. Possibly,

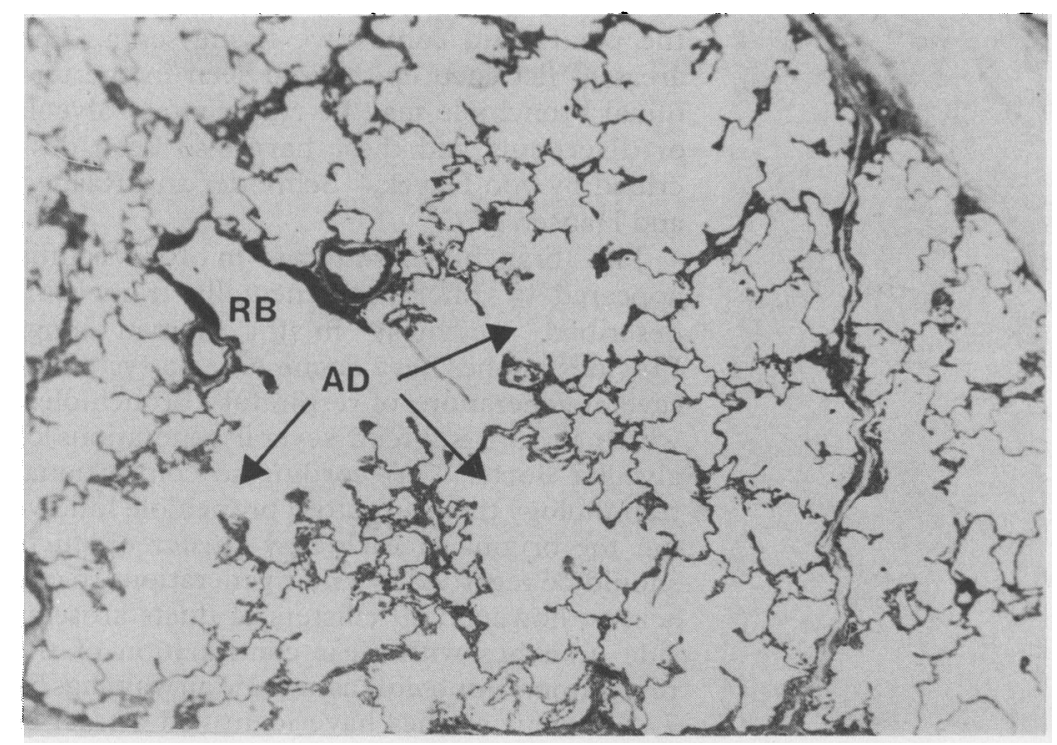

Figure 5 First branches (alveolar ducts) arising from the respiratory bronchiole supplying the right hemiacinus. $R B-$ respiratory bronchiole; $A D-$ alveolar ducts.

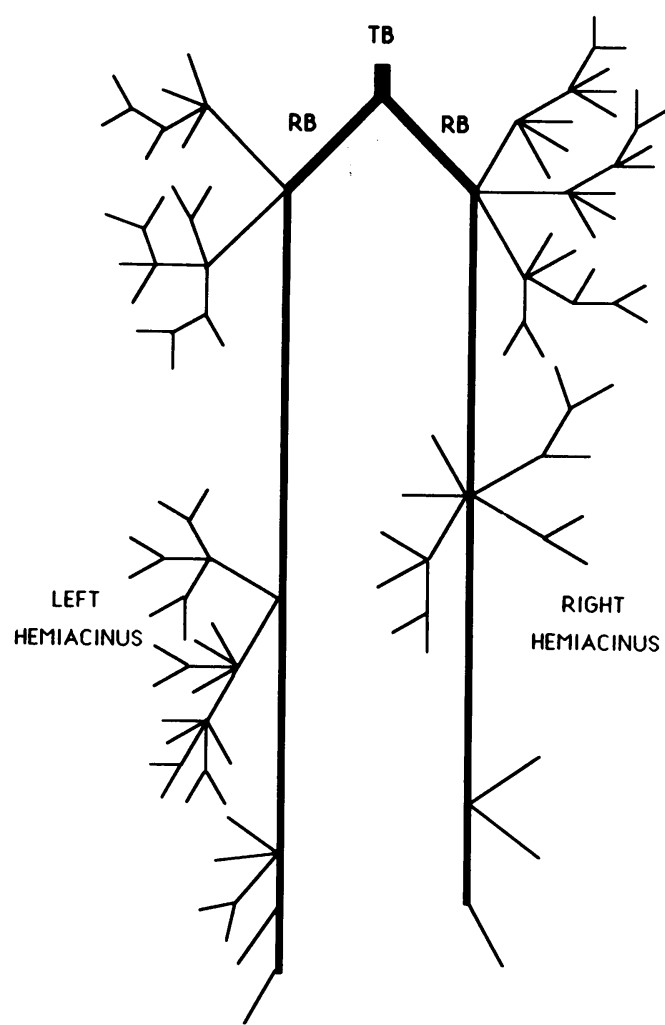

Figure 4 Schematic representation of the overall structure of the acinus. Length and directions of branches not to scale. Terminal bronchiole; -respiratory bronchiole; -alveolar duct.

however, final branches in both the left and the right hemiacinus close to the pleural surface were missed because of our inability to cut right up to the extreme end of the acinus.

Although the respiratory bronchioles subtended approximately equal numbers of alveolar ducts ( 51 for the right and 58 for the left), the alveolar duct systems were asymmetric with different numbers of branches at each level. The alveolar duct systems became progressively less complex towards the periphery as the acinus narrowed approaching the edge of the lung.

The total volume of the acinus was estimated to be $153 \mathrm{~mm}^{3}$ with a small additional volume close to the edge of the lung.

\section{Discussion}

An initial problem in this study was obtaining an appropriate lung free of trauma or disease. Although it was not known whether the accident victim had been a smoker, his young age precluded the possibility of a major degree of smoking induced lung disease. The normal chest radiograph before death and the normal lung weight reassured us that there was no lung trauma or pulmonary oedema.

There were several reasons for reconstructing an acinus from the periphery of the lung. Here were found connective tissue septa between the acini, which facilitated reconstruction because there were fewer interdigitations with neighbouring acini. We thought that it would be of interest to look at an acinus that had developed in a part of the lung where space 


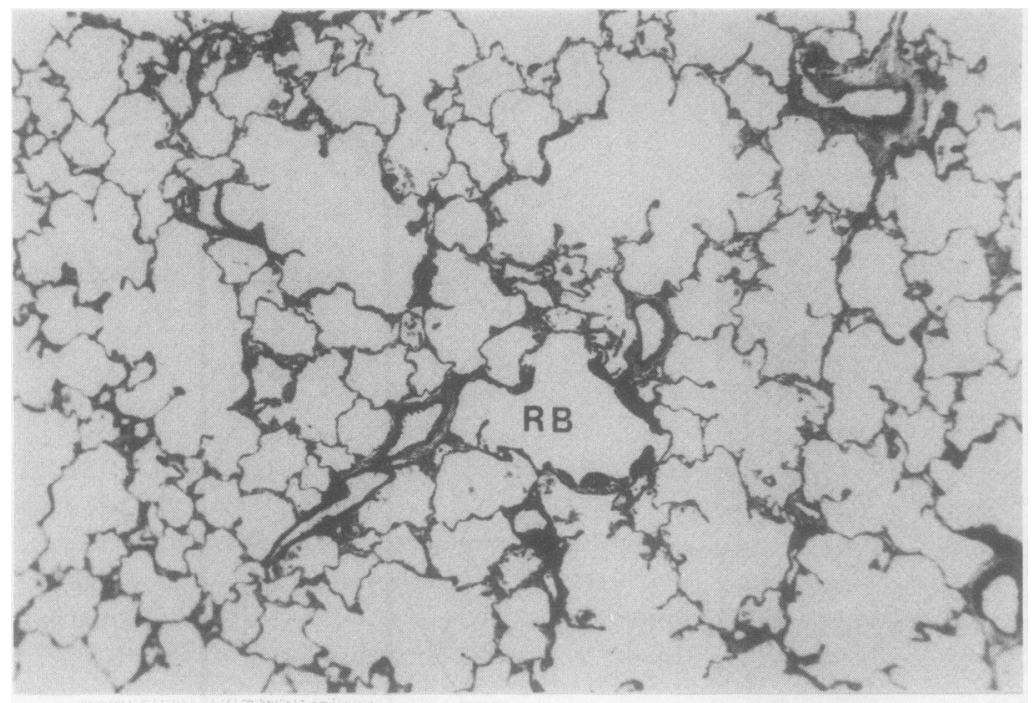

Figure 6 Respiratory bronchiole between first and second alveolar duct systems. $R B$-respiratory bronchiole.

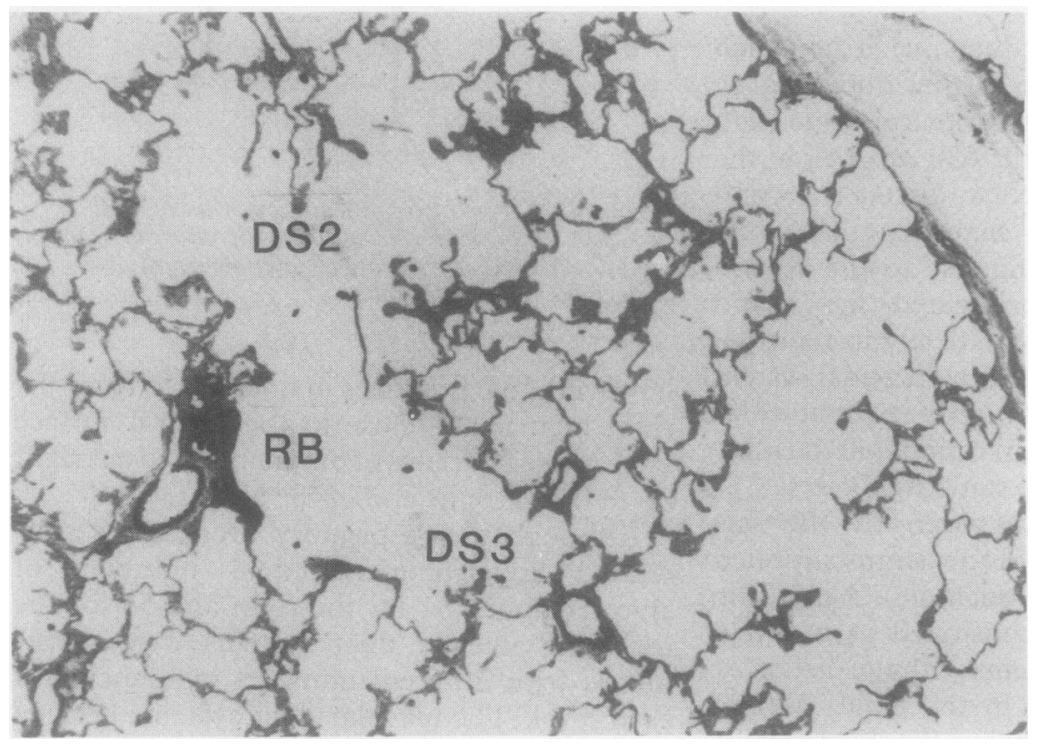

Figure 7 Second and third duct systems (DS) arising from opposite sides of the respiratory bronchiole $(R B)$.

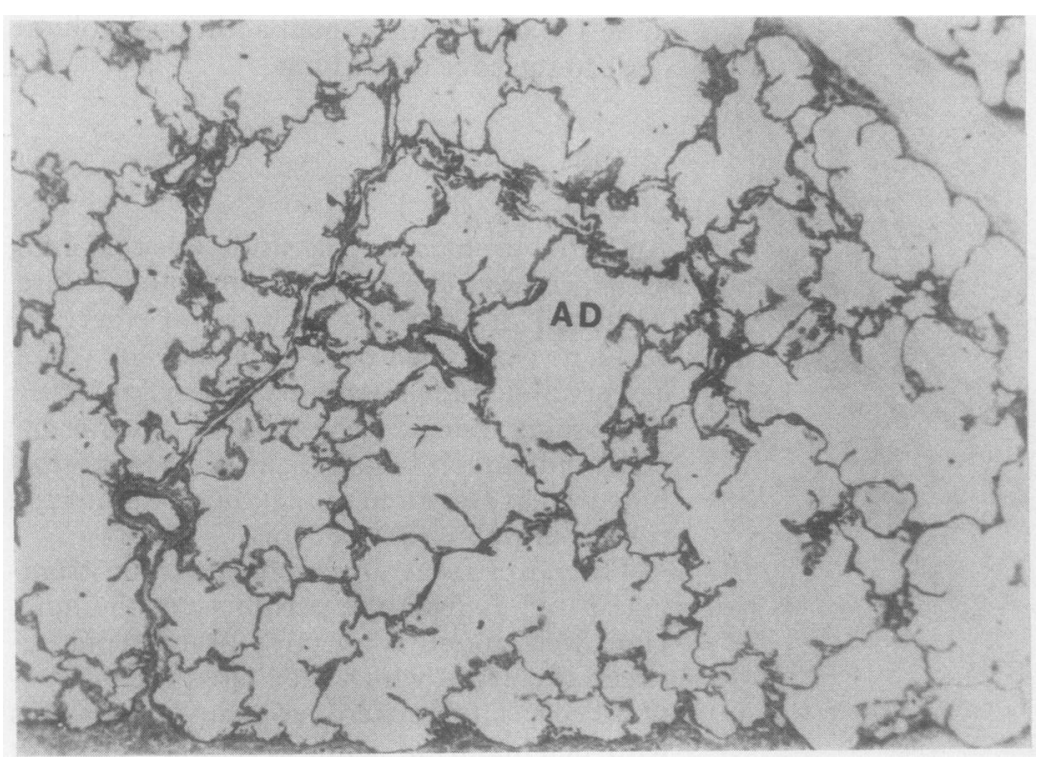

Figure 8 Respiratory bronchiole supplying the right hemiacinus terminating in a fully alveolated state - that is, becoming an alveolar duct. was constrained because this may have affected development of airway branching.

Two major technical difficulties were encountered. Firstly, paraffin embedded blocks yielded sections with variable shrinkage and distortion, which made accurate stacking of sections impossible. We therefore decided to embed the tissue blocks in plastic. This, however, proved to be technically very difficult. After considerable trial and error we developed a method that produced good tissue penetration of the embedding medium and a block free of air bubbles. Unfortunately, the tissue was very close to the edge of the block and therefore sections could not be cut right up to the extreme distal end of the acinus. The amount of tissue lost was less than $1 \mathrm{~mm}$ but further distal branches may have been missed.

The second major problem was related to the determination of the branching patterns of the alveolar ducts. Sections $25 \mu \mathrm{m}$ apart were photographed and enlarged and these were initially examined to detect alveolar ducts arising from the respiratory bronchioles. Each of these ducts was then followed by examining successive photographs moving either proximally or distally, as appropriate. The same process was followed for each generation of alveolar ducts and this proved to be very difficult and time consuming. On occasion we had to refer to additional sections under the microscope within the $25 \mu \mathrm{m}$ intervals between photographs to be certain of the branching patterns. Visualisation of the three dimensional reconstruction was of value only for small parts of the acinus as the whole structure is too complex, a problem also encountered by Mercer and Crapo, ${ }^{10}$ who performed a three dimensional reconstruction of a rat acinus.

An unexpected finding that facilitated this work was the enclosure of the acinus by the pleural surfaces and connective tissue septa. In the human lung there are usually interlobular septa, though these are often incomplete, and rarely interacinar septa. ${ }^{911}$ In the acinus described in this paper the only interacinar interdigitations were on the proximal boundary, the lateral and distal boundaries being defined by the pleura and connective tissue septa. The areas of flattened epithelium seen in the terminal bronchiole may be rudimentary alveoli or diverticula and these have also been described by von Hayek, ${ }^{11}$ Schreider and Raabe, and Hansen et al. ${ }^{8}$

The branching patterns in this acinus appeared to differ fundamentally from acini described previously in the human lung. Previous studies have documented a system of several generations of respiratory bronchioles, which in turn subtend several generations of alveolar ducts. ${ }^{37-9}$ According to conventional terminology the respiratory bronchiole following the origins of each new cluster of ducts should be regarded as a new generation. In our acinus, however, all clusters of ducts arose as side branches with clear continuation of the respiratory bronchiole as a single airway (figs 5 7). Previous studies have identified a total of nine to twelve generations of intra-acinar airways, the branching pattern being mainly 
(irregularly) dichotomous with some trichotomous and quadrivial divisions. In their recent study Haefeli-Bleuer and Weibel, ${ }^{9}$ using a silicone rubber cast of an acinus, considered divisions to be mainly dichotomous with polychotomous branches really being dichotomous branches in close proximity. Although polychotomous branches appeared common in the present study we could not always be certain that these were not dichotomous branches in close succession. Following each cluster of alveolar ducts, which usually arose from a multiple origin, the respiratory bronchiole continued as a single airway and finally became a duct. Because of the wedge shape of the edge of the lung and the pleural boundaries the alveolar duct systems became smaller and less complex towards the periphery of the acinus. The total acinar volume $\left(153 \mathrm{~mm}^{3}\right)$ was within the range previously described $\left(50-450 \mathrm{~mm}^{3}\right){ }^{9}$ An intraacinar duct to duct communication and bronchiole-duct communication were identified but others are likely to have been missed. Such communications would be much more obvious with an acinar cast than with a reconstruction technique. No interacinar pathways for collateral ventilation were identified.

The difference between this acinus and others described in the human lung may be related to differences of position within the lung from which the acinus was derived. The acinus described in the lungs of a child by Boyden, ${ }^{3}$ however, was derived from a location similar to that of ours but had the standard acinar structure described by others.

1 Rodriguez M, Ber S, Farre A, Weibel ER. Pulmonary acinus: geometry and morphometry of the peripheral airway system in rat and rabbit. Am J Anat 1987;180: 143-55

2 Pump KK. Morphology of the acinus of the human lung. Dis Chest 1969;56:126-34.

3 Boyden EA. The structure of the pulmonary acinus in a child of six years and eight months. Am J Anat 1971;132:275300.

4 Hansen JE, Ampaya EP. Human air space shapes, sizes, areas and volumes. J Appl Physiol 1975;38:990-5.

5 Parker H, Horsfield K, Cumming G. Morphometry of distal airways in the human lung. J Appl Physiol 1971;31: 386-91.

6 Horsfield K, Cumming G. Morphology of the bronchial tree in man. J Appl Physiol 1968;24:373-83.

7 Schreider JP, Raabe OG. Structure of the human respiratory acinus. Am J Anat 1981;162:221-32.

8 Hansen JE, Ampaya EP, Bryant GH, Navin JJ. Branching pattern of airways and air spaces of a single human terminal bronchiole. J Appl Physiol 1975;38:983-9.

9 Haefeli-Bleuer B, Weibel ER. Morphometry of the human pulmonary acinus. Anat Record 1988;220:401-14

10 Mercer RR, Crapo JD. Three dimensional reconstruction of the rat acinus. J Appl Physiol 1987;63:785-94.

11 Von Hayek H. The human lung. New York: Hefner, 1960: 172-3. 